\title{
A Mitogen-Activated Protein Kinase Gene (MGV1) in Fusarium graminearum Is Required for Female Fertility, Heterokaryon Formation, and Plant Infection
}

\author{
Zhanming Hou, ${ }^{1}$ Chaoyang Xue, ${ }^{1}$ Youliang Peng, ${ }^{1}$ Talma Katan, ${ }^{2}$ H. Corby Kistler, ${ }^{3}$ and Jin-Rong Xu ${ }^{1}$ \\ ${ }^{1}$ Department of Botany and Plant Pathology, Purdue University, West Lafayette, IN 47907, U.S.A.; ${ }^{2}$ Department of Plant \\ Pathology, The Volcani Center, Bet Dagan 50250, Israel; ${ }^{3}$ USDA-ARS, Department of Plant Pathology, University of Minne- \\ sota, St. Paul, MN 55108, U.S.A.
}

Received 7 May 2002. Accepted 20 July 2002.

Fusarium graminearum is an important pathogen of small grains and maize in many areas of the world. Infected grains are often contaminated with mycotoxins harmful to humans and animals. During the past decade, $F$. graminearum has caused several severe epidemics of head scab in wheat and barley. In order to understand molecular mechanisms regulating fungal development and pathogenicity in this pathogen, we isolated and characterized a MAP kinase gene, MGV1, which is highly homologous to the MPS1 gene in Magnaporthe grisea. The MGV1 gene was dispensable for conidiation in $F$. graminearum but essential for female fertility during sexual reproduction. Vegetative growth of mgv1 deletion mutants was normal in liquid media but reduced on solid media. Mycelia of the mgvl mutants had weak cell walls and were hypersensitive to cell wall degrading enzymes. Interestingly, the mgv1 mutants were selfincompatible when tested for heterokaryon formation, and their virulence was substantially reduced. The ability of the mutants to accumulate trichothecene mycotoxins on inoculated wheat was also greatly reduced. Our data suggest that MGVI in F. graminearum is involved in multiple developmental processes related to sexual reproduction, plant infection, and cell wall integrity.

Additional keywords: fungal pathogenicity, Gibberella zeae

Fusarium head blight (scab) is a disease of wheat and barley that can cause reduction in both crop yield and grain quality and has emerged in the past 10 years as an important constraint on small grain production (Windels 2000). In North America, the primary pathogen causing this disease is Fusarium graminearum Schwabe, lineage 7 (O'Donnell et al. 2000) (teleomorph Gibberella zeae). Infected grains are often contaminated with mycotoxins, such as deoxynivalenol (DON) and zearalenone, which are harmful to humans and animals (Desjardins et al. 1993; Windels 2000). In addition to being an important pathogen of wheat and barley, $F$. graminearum is also associated with stalk and ear rots of maize (Sutton 1982).

The development of ascospores is a vital process in the life cycle of the fungus and in disease development. The fungus overwinters on infected plant debris as saprophytic mycelia and produces spores when weather conditions favor fungal development in the spring. Ascospores and conidia formed by $F$.

Corresponding author: Jin-Rong Xu; Telephone: 765-496-6918; Fax: 765-494-0363; E-mail: xu@btny.purdue.edu graminearum are believed to be the main inoculum infecting flowering wheat heads (Francl et al. 1999; McMullen et al. 1997). Ascospores are forcibly discharged from mature perithecia (Trail et al. 2002) formed on the surface of debris and possibly are dispersed by wind (Parry et al. 1995). Conidia may be dispersed primarily by rain splash and are important in spreading the disease in the field (Sutton 1982) and colonizing plant vegetative tissues, which serve as the substrate for overwintering mycelia (Wearing and Burgess 1977). F. graminearum is a homothallic ascomycete that can be readily forced to outcross (Bowden and Leslie 1999) and is amenable to classical and molecular genetic techniques such as fungal transformation and gene disruption (Proctor et al. 1995). However, there are few molecular studies of the development and pathogenicity of this important pathogen. The TRI5 gene, involved in trichothecene production, is the only virulence gene characterized in F. graminearum (Proctor et al. 1995).

Signaling pathways involved in plant infection by the rice blast fungus, Magnaporthe grisea, have been well studied, and three mitogen-activated protein (MAP) kinases have been characterized (Xu 2000). The MAP kinase encoded by MPS1, a homologue of Saccharomyces cerevisiae SLT2, is important for maintaining cell wall integrity and essential for plant penetration (Xu et al. 1998). Appressoria formed by M. grisea mps 1 mutants neither penetrate plant cells nor elicit plant defense responses. Conidiation and aerial hyphae development are drastically reduced in mps 1 mutants, which are also female-sterile during sexual reproduction ( $\mathrm{Xu}$ et al. 1998). Homozygous mutants of the human pathogen Candida albicans disrupted in $M K C 1$, an MPS1 homologue, produce less invasive growth on Spider medium and are less virulent in an experimental infection system using a murine model (Diez-Orejas et al. 1997). The MPS1 and MKC1 MAP kinase genes have $80 \%$ similarity, and both are involved in fungal pathogenesis.

In this study, we isolated and characterized the $F$. graminearum gene $M G V 1$ (map kinase for growth and virulence 1), a homologue of MPS1. F. graminearum does not form appressoria or other specialized penetration structures for plant infection, but ascospores and conidia are important in disease development and dispersal, and the production of these spores may be regulated by $M G V 1$. Site-directed $m g v 1$ deletion mutants had reduced virulence and failed to produce perithecia and ascospores. However, hyphal growth and conidiation of the $m g v l$ mutants in liquid culture were not affected, although their growth rate was reduced when they were cultured on solid media, and their mycelia appeared to have defective cell walls. 


\section{RESULTS}

MGV1 encodes a MAP kinase homologous to M. grisea MPS1.

The polymerase chain reaction (PCR) product amplified with primers $\mathrm{M} 3 \mathrm{~K} 1$ and $\mathrm{M} 3 \mathrm{~K} 2$ was cloned and sequenced. One PCR clone, pZMH2, was found to be homologous to MPS1 by BLASTX searches and was used to screen $F$. graminearum cosmid and cDNA libraries. Sequence analysis of the corresponding cosmid and cDNA clones revealed an open reading frame (ORF) encoding a protein with 416 amino acids that is homologous to those encoded by MPS1 and other fungal MAP kinase genes (Fig. 1). The predicted amino acid sequence of the $M G V 1$ gene is $86 \%$ identical and $92 \%$ similar to that of M. grisea MPS1 and $51.2 \%$ identical and $76.9 \%$ similar to that of $S$. cerevisiae SLT2 (Lee et al. 1993). Southern blot analysis showed that $M G V 1$ is a single-copy gene in $F$. graminearum (data not shown). The $M G V 1$ coding region contains all conserved protein kinase subdomains and the characteristic MAP kinase dual phosphorylation sites TEY (residues 174-176). Phylogenetic analysis indicated that MGV1 is most similar to MPS1, and both MPS1 and MGV1 are in the same clade with the $S$. cerevisiae SLT2 (Xu 2000).
Isolation of $m g v 1$ deletion mutants by gene replacement.

The $M G V 1$ gene replacement vector pZMH6 was constructed by replacing the first $680 \mathrm{bp}$ of the $M G V 1$ ORF and $770 \mathrm{bp}$ of its upstream region with the $h p h$ gene (Fig. 2A). Over 100 hygromycin-resistant transformants were isolated and screened with PCR primers $17 \mathrm{~F}$ and H855R (data not shown). Two putative $m g v l$ deletion mutants were identified and confirmed by Southern blot analysis (Fig. 2B). When hybridized with a fragment amplified with primers $13 \mathrm{~F}$ and $\mathrm{K} 1 \mathrm{R}$ as the probe (Fig. 2B), only the wild-type $\mathrm{PH}-1$ and a randomly selected ectopic transformant, N47, had the 1-kb HindIII band. The mgvl deletion transformants, C6 and N35, did not have any hybridization signal, because the probe sequence was deleted in pZMH6. When the same blot was stripped and rehybridized with the hph gene as the probe, C6, N35, and N47, but not PH-1, had distinct hybridization bands. Only a single $7.5-\mathrm{kb}$ band in $\mathrm{C} 6$ hybridized to $h p h$, but multiple bands hybridized in N35, indicating that both homologous recombination and multiple ectopic integrations of pZMH6 occurred in this transformant. The deletion of the MGV1 gene in C6 and N35 was further confirmed by Southern blot analysis with DNA samples digested with KpnI (data not shown). Even though N35 was identical to C6 in colony morphology, to avoid unexpected interference

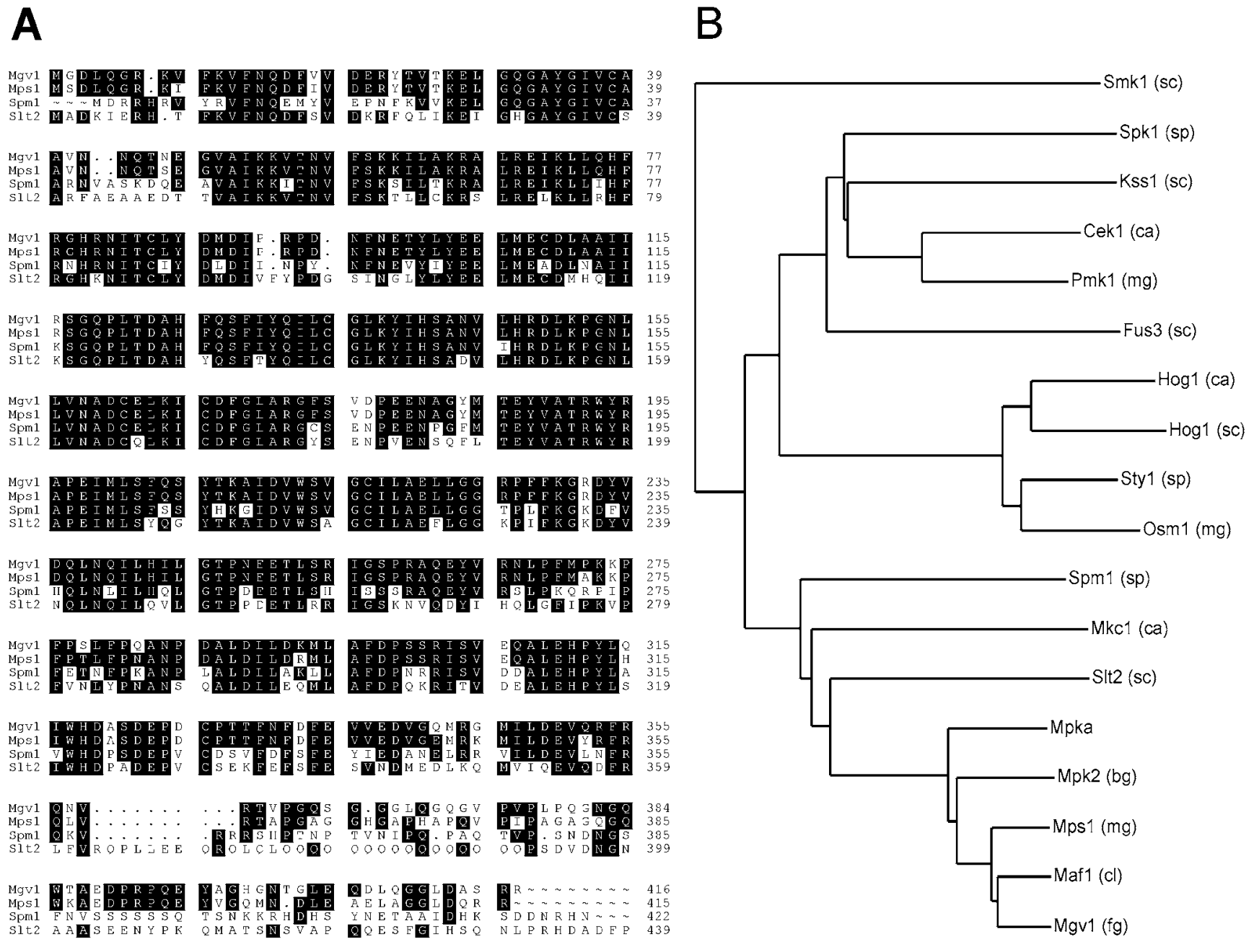

Fig. 1. MGV1 encodes a mitogen-activated protein (MAP) kinase in Fusarium graminearum. A, Alignment of the predicted amino acid sequence encoded by MGV1 with those of its homologues. The GenBank accession numbers are F. graminearum Mgv1 (AF492766), Magnaporthe grisea Mps1 (AF02316), Colletotrichum lagenarium Maf1 (AY064246), Blumeria graminis Mpk2 (AF301165), Aspergillus nidulans MpkA (U59214), Schizosaccharomyces pombe Spm1 (U65405), and Saccharomyces cerevisiae Slt2 (X59262). B, Phylogenetic comparison of MGV1 MAP kinase with other fungal MAP kinases. The amino acid sequences of MAP kinases from S. cerevisiae (sc), S. pombe (sp), Candida albicans (ca), M. grisea (mg) and other fungi were analyzed by the Clustal X program (Thompson et al. 1994) to create a progressive alignment dendrogram. The branch lengths are proportional to the mean number of differences per residue along each branch. Mps1 and Mgv1 cluster together with the Slt2 MAP kinase from S. cerevisiae. 
resulting from the ectopic integration of pZMH6 in N35, only C6 was used in most of the experiments for characterizing the phenotypes of the $m g v l$ mutants.

\section{Reduced vegetative growth of $m g v 1$ mutants on solid media but not in liquid media.}

On V8 juice agar plates, colonies of the $m g v l$ mutants were smaller and more compact than those of the wild-type strain PH-1 and often had uneven margins (Fig. 2C). While the wildtype strain produced abundant aerial hyphae on V8 juice agar, the mgvl mutants produced fewer and shorter aerial hyphae (Fig. 2C). Aerial hyphae produced by the mgvl mutants also had less pigment. One-week-old colonies on V8 juice agar plates were whitish, in contrast to the pinkish colonies formed by $\mathrm{PH}-1$. After incubation on V8 juice medium for 5 days, the average diameters of $\mathrm{PH}-1$ and $\mathrm{C} 6$ colonies were 7.7 and 2.3 $\mathrm{cm}$, respectively (Table 1 ). Vegetative growth of the $m g v 1 \mathrm{mu}-$

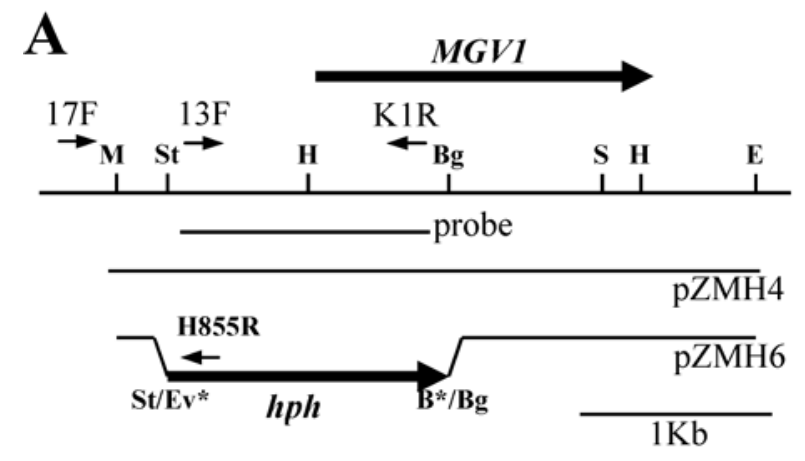

B
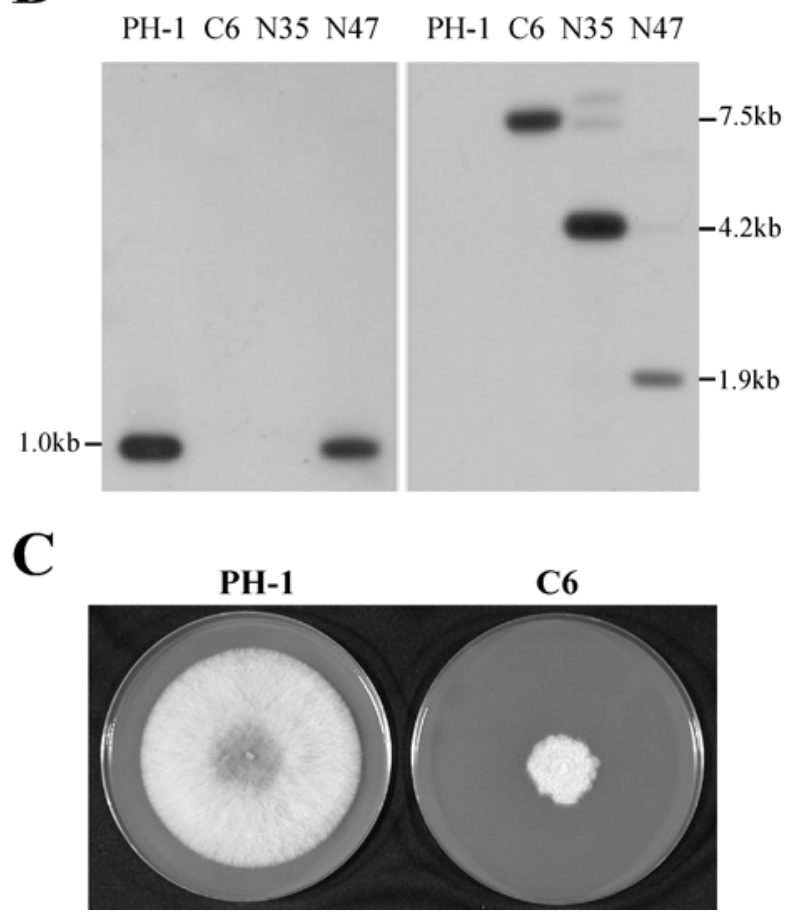

Fig. 2. Fusarium graminearum $M G V 1$ gene replacement construct and mutants. A, Physical map of the MGV1 genomic region and gene replacement vector pZMH6. Arrows indicate the orientation of the MGV1 and $h p h$ genes. The restriction enzymes are $B g l \mathrm{II}(\mathrm{Bg}), E c o$ RI $(\mathrm{E})$, HindIII (H), MboI (M), SalI (S), and StuI (St). B, Southern blot of wildtype $F$. graminearum $\mathrm{PH}-1$, the ectopic transformant $\mathrm{N} 47$, and the $m g v 1$ mutants C6 and N35. All DNA samples were digested with HindIII. The blot was hybridized with the fragment amplified with $13 \mathrm{~F}$ and $\mathrm{K} 1 \mathrm{R}$ as the probe. C, Colony morphology of PH-1 and mgv1 mutant C6 cultured on V8 juice agar plates. The photograph was taken after 3 days of incubation at $25^{\circ} \mathrm{C}$. tant C6 was also significantly reduced when it was cultured on $\mathrm{CM}$ and $5 \times$ YEG media (Table 1), indicating that the reduction in growth rate was not related to the specific media used. Similarly, different media had no effect on the production of aerial hyphae and pigment in the mgvl mutants, because C6 produced whitish compact colonies on all media tested.

We also determined hyphal growth of an mgvl mutant in liquid CMC medium by measuring the fungal biomass accumulated after incubation for 3 days. No significant differences were observed between PH-1, C6, and N47 (Table 1). When cultured in nutritionally rich yeast extract-peptone-dextrose (YEPD) medium, both the wild type and the $m g v l$ mutant produced more abundant vegetative mycelia. However, there was no significant difference in fungal biomass accumulation between the wild-type strain and the mgvl mutant (data not shown). Thus, the mgvl deletion mutant had no defect in vegetative growth in liquid media.

\section{Conidiation in mgv1 mutants.}

On V8 juice agar plates, both $\mathrm{PH}-1$ and $\mathrm{C} 6$ produced conidia, but not efficiently. Because vegetative growth of the mgv1 mutant on V8 juice agar plates was reduced, quantitative comparison of the conidiation of $\mathrm{C} 6$ and $\mathrm{PH}-1$ colonies was difficult. In liquid CMC medium, the $m g v l$ mutant produced abundant conidia (Fig. 3A). On average, PH-1, N47, and C6 all produced about $1.5 \times 10^{5}$ conidia/ml after 5 days of incubation (Fig. 3A), indicating that the deletion of $M G V 1$ had no obvious effect on conidiation.

Conidia produced by the mgvl mutant C6 were normal in size and morphology. No defect was observed in the germination of conidia of C6 and N35 (data not shown). Old cultures of wild-type $F$. graminearum strains produce a few thickwalled cells within conidia. These cells do not resemble the globose chlamydospores, which are formed only rarely in $F$. graminearum culture and have been described by many authorities (Booth 1971; Gerlach and Nirenberg 1982; Nelson et al. 1983). The mgvl mutant was also able to form thick-walled cells within conidia in CMC cultures more than 7 days old (Fig. 3B). However, the number of C6 and N35 conidia that developed wall thickenings was less than $5 \%$ of the number of PH-1 and N47 conidia that developed them (data not shown). In a small portion (less than $0.1 \%$ ) of the C6 conidia having wall thickenings, the apical and basal cells of macroconidia were disarticulated, resulting in spore fragmentation (Fig. 3B). These fragmented conidia germinated normally when transferred to $\mathrm{CM}$ or $5 \times$ YEG media (data not shown). No fragmented spores were observed in macroconidia produced by PH-1 and N47 cultures grown under the same conditions. Most likely conidia with wall thickenings also have weakened cellto-cell junctions as a result of the $m g v l$ deletion.

\section{mgv1 mutants are female-sterile.}

To determine the potential function of $M G V 1$ in sexual reproduction, the $m g v 1$ mutants were assayed for the production of perithecia on carrot agar medium under conditions that would

Table 1. Vegetative growth of Fusarium graminearum strains on different media

\begin{tabular}{|c|c|c|c|c|}
\hline \multirow[b]{2}{*}{ Strain } & \multicolumn{3}{|c|}{ Colony diameter $(\mathbf{c m})^{\mathrm{a}}$ on: } & \multirow{2}{*}{$\begin{array}{c}\text { Dry weight } \\
\text { (mg) }^{\mathrm{b}}\end{array}$} \\
\hline & V8 juice & $5 \times$ YEG & $\mathbf{C M}$ & \\
\hline PH-1 ( & $7.7 \pm 0.2$ & & 2 & 3 \\
\hline-0 & & & 3.6 & \\
\hline N47 (ectopi & $8.4 \pm 0.1$ & $6.6 \pm 0.1$ & $8.6 \pm 0.4$ & $140.5 \pm 8.8$ \\
\hline
\end{tabular}

${ }^{a}$ Colony diameter was measured after incubation for 5 days. The mean and error were calculated from at least three independent experiments.

${ }^{\mathrm{b}}$ Dry weight of fungal biomass grown in liquid CMC medium. 
promote selfing or outcrossing. As a homothallic fungus, both the wild-type PH-1 and the ectopic transformant N47 alone produced abundant perithecia after 2-3 weeks. However, the mgv1 mutant C6 failed to produce any perithecia under selfing conditions, even after 1 month of incubation (Fig. 4A). No perithecia or protoperithecia were observed in cultures of C6 (Fig. $4 \mathrm{~A}$ ) or N35, indicating that autonomous sexual reproduction was abolished in the mgvl mutants.

On outcrossing plates with the mgvl mutant C6 or N35 and the PH-1 nitl mutant 11622 growing side-by-side, perithecia were observed only on the nit1 mutant colonies (Fig. 4B). No perithecia were observed on the C6 or N35 colonies 3 weeks or more after cross-fertilization (Fig. 4B). Random ascospores collected from perithecia formed on the nit 1 mutant colonies in C6 $\times 11622$ outcross plates were cultured on minimal medium containing hygromycin $(250 \mu \mathrm{g} / \mathrm{ml})$ and $1 \%$ potassium chlorate. All 20 randomly selected ascospore progeny that grew on this medium had the colony morphology of $m g v l$ mutants and

A

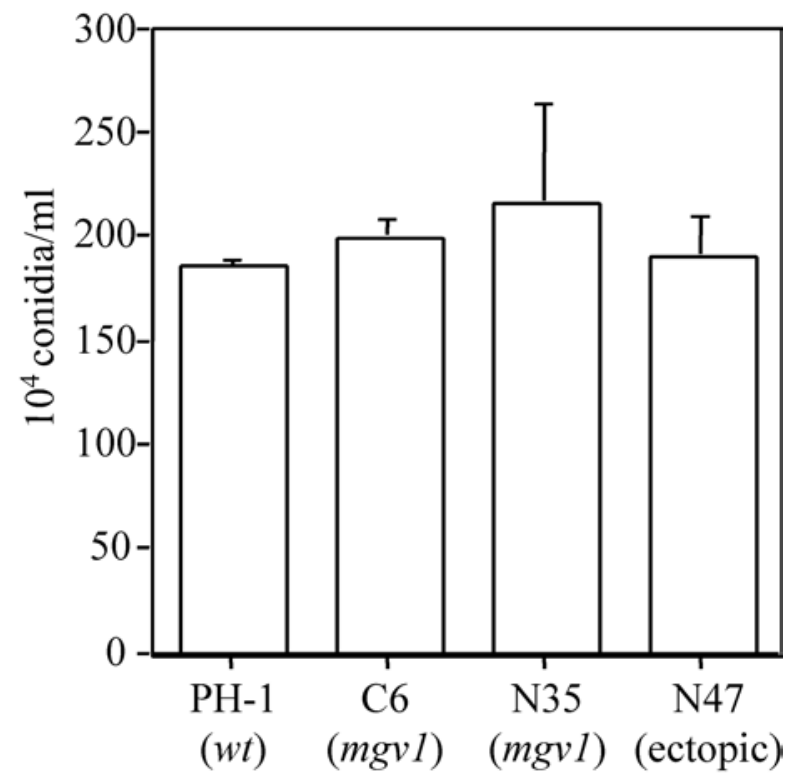

B
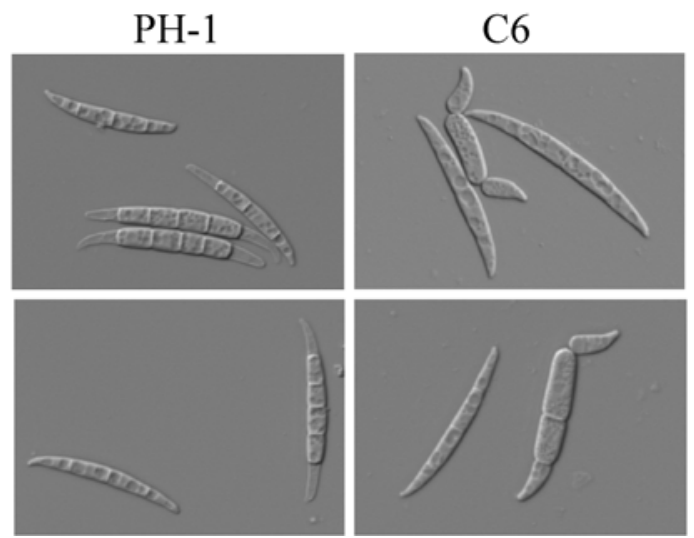

Fig. 3. Conidia produced by $m g v 1$ deletion mutants of Fusarium graminearum. A, Conidiation in liquid CMC medium. Concentrations of conidia produced by cultures of the wild-type strain PH-1, mgv1 mutants C6 and N35, and the ectopic transformant N47 were determined after incubation for 5 days. Means and standard errors were calculated from three independent experiments. B, Morphology of conidia and thickwalled conidia produced by PH-1 and mgv1 mutant C6. A small portion of conidia became fragmented in C6 cultures more than 7 days old. were confirmed by Southern blot analysis to be $m g v l$ mutants (data not shown). Because they were resistant to both hygromycin and potassium chlorate, we conclude that these recombinant colonies were from outcrosses between the hygromycinresistant $m g v 1$ and the chlorate-resistant nitl mutants. Thus, the $m g v l$ deletion mutant was unable to form the female fruiting structure on it own (female-sterile) but was still able to contribute to a sexual outcross (male-fertile). All of the 20 hygromycin-sensitive progeny isolated from the same outcross grew at the same rate as $\mathrm{PH}-1$ and formed normal colonies with abundant aerial hyphae, indicating that the hygromycin-resistant marker co-segregated with the colony phenotype observed in the $m g v l$ deletion mutants.

\section{Cell wall defects in mgv1 mutants.}

Because the central part of $m g v l$ colonies sometimes underwent autolysis in cultures more than 3-4 weeks old, we suspected that $m g v l$ mutants had defects in hyphal cell walls. To confirm this, we treated $F$. graminearum germlings collected from conidia germinated in liquid YEPD medium for $14 \mathrm{~h}$ with the cell wall degrading enzyme Driselase. After incubation at $30^{\circ} \mathrm{C}$ for only $30 \mathrm{~min}$, few protoplasts were observed among the wild-type germlings, while germlings from $m g v 1$ mutant C6 were well digested and released abundant protoplasts (Fig. 5A).

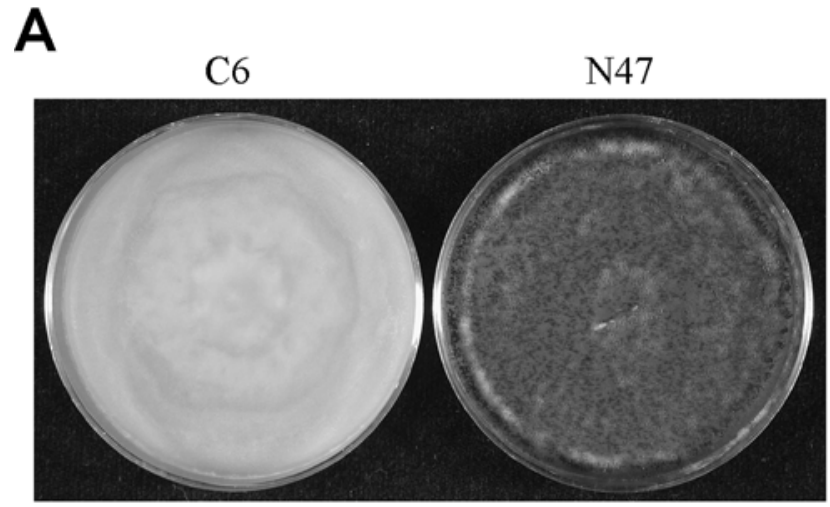

B

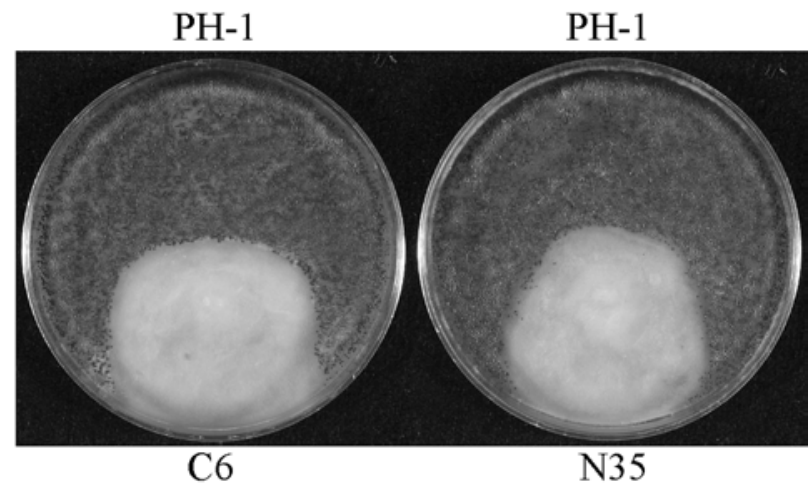

Fig. 4. Sexual reproduction in mgv1 mutants of Fusarium graminearum. A, The mgv1 mutants were self-sterile. Abundant perithecia (black dots) were produced by the ectopic transformant N47 (right) 2 weeks after fertilization on carrot agar plates. The $m g v 1$ mutant C6 (left) failed to form any perithecia under the same conditions. B, Outcrossing between the wild-type strain PH-1 (in the upper part of the plates) and the $m g v 1$ deletion mutants C6 and N35 (in the lower part of the plates). Only the colonies of PH-1, and not those of $\mathrm{C} 6$ and N35, developed perithecia on co-inoculated carrot agar plates. The photographs were taken 2 weeks after cross-fertilization. 
When conidia germinated in liquid CMC medium for $18 \mathrm{~h}$ at $25^{\circ} \mathrm{C}$ were shifted to $32^{\circ} \mathrm{C}$ and incubated for another $12 \mathrm{~h}$, terminal and intercalary swollen bodies developed in many germlings from the mgvl mutants C6 and N35 (Fig. 5). When 18-h germlings were shifted to $37^{\circ} \mathrm{C}$ and incubated for over $12 \mathrm{~h}$, most of the mgvl germ tubes had swollen bodies, and many had burst. We also observed that incubation temperatures higher than $32^{\circ} \mathrm{C}$ significantly inhibited the formation of conidia in the mgvl mutants (data not shown). These observations suggest that the $m g v l$ mutants have an impaired cell wall and are sensitive to elevated incubation temperatures (above $32^{\circ} \mathrm{C}$ ). This defect in cell wall integrity appeared to be more severe when the $m g v l$ mutants were cultured on nutritionally rich media, such as 5xYEG and TB3. Intercalary and apical hyphal swollen bodies could be observed in some mycelia even when the mutants were cultured at $25^{\circ} \mathrm{C}$ in these media (data not shown).

\section{Virulence is reduced in mgv1 mutants.}

To determine whether the virulence of the mgvl mutants is affected, flowering wheat heads were inoculated with conidia from PH-1, C6, N35, and N47. Most of the basal spikelets inoculated with $m g v l$ spore suspensions developed typical scab symptoms 14 days after inoculation, similar to those inoculated with conidia from PH-1 and N47 (Fig. 6). However, the number of uninoculated spikelets that developed scab symptoms on wheat heads basally inoculated with the mgvl mutant C6 or N35 was significantly reduced (Table 2). At 14 days after inoculation, only one or two spikelets were blighted on most wheat heads inoculated with the mgvl mutants. Under the same conditions, about half of the spikelets were blighted on wheat heads inoculated with PH-1 or N47 (Fig. 6). These data indicate that the ability of the $m g v l$ mutants to colonize and spread in flowering wheat heads was reduced.

To determine whether the reductions in virulence and growth rate on solid media were directly related to the deletion of $M G V 1$, a 9-kb BamHI fragment containing the wild-type $M G V 1$ gene was cloned in pBCSK and introduced into $m g v l$ mutant C6 by co-transformation with pSM334. Neomycinresistant transformants were screened by PCR with primers $13 \mathrm{~F}$ and $\mathrm{K} 1 \mathrm{R}$, and the presence of the wild-type $M G V 1$ allele was confirmed by Southern blot analysis. Complemented $M G V 1$ transformants grew at rates comparable to that of the wild-type strain PH-1 (Table 1) and had normal virulence on flowering wheat heads (data not shown). Thus, reintroduction of the wild-type $M G V 1$ allele restored the growth and virulence defect in the mgvl mutants.

\section{Production of DON in mgv1 mutants.}

To date, DON is the only known virulence factor in $F$. graminearum (Proctor et al. 1995). To determine whether DON accumulation in plants is affected by the $m g v l$ deletion, spikelets of the wheat cultivar Norm were inoculated with N35, C6, or the ectopic transformant N47. The trichothecene content of

Table 2. Spread of head scab symptoms from basal spikelets inoculated with various strains of Fusarium graminearum

\begin{tabular}{lcc}
\hline Strain & $\begin{array}{c}\text { No. of heads } \\
\text { inoculated }\end{array}$ & Disease index $^{\mathbf{a}}$ \\
\hline C6 (mgv1 mutant) & 33 & $1.97 \pm 1.26$ \\
N35 (mgv1 mutant) & 31 & $1.81 \pm 1.54$ \\
N47 (ectopic) & 32 & $5.56 \pm 2.00$ \\
PH-1 (wild type) & 35 & $7.09 \pm 2.86$ \\
\hline
\end{tabular}

${ }^{\text {a }}$ Disease was rated by the number of symptomatic spikelets 14 days after inoculation of a basal spikelet. C6 and N35 are significantly less virulent than either $\mathrm{N} 47$ or PH-1 $(p=0.0001)$. the plants was measured 2 weeks after inoculation. While DON and 15-acetyl-DON accumulated to levels reaching above 200 ppm in samples inoculated with N47, trichothecene concentrations in samples inoculated with the mgvl mutants N35 and C6 were less than $1.5 \mathrm{ppm}$ or not detectable (Table 3 ).

\section{mgv1 mutants are self-incompatible in heterokaryon formation.}

To determine whether $m g v l$ mutants are capable of normal cell fusion and heterokaryon formation, several independent auxotrophic (nitl) mutants were made from mgvl strains C6 and N35 and then paired on minimal medium with a PH-1 strain having a nonallelic auxotrophic mutation (nitM) also in the pathway for nitrate reduction. As a control, nitl mutations were made from the ectopic transformant strain N47 and also paired with the PH-1 nitM strain. Wild-type growth was readily observed (Fig. 7) at the convergence of colonies of N47 (MGV1 nitl) and PH-1 (MGVI nitM), indicating successful cell fusion and heterokaryon formation. Repeated pairing of independent C6 (mgvl nit l) and N35 (mgvl nit 1) strains with PH-1 (MGV1 nitM) never resulted in wild-type growth at the convergence of the colonies (Fig. 7). When samples were examined under a microscope, hyphal fusion was not observed in the mgvl mutants C6 and N35 (data not shown). Thus, while the ectopic transformant N47 maintained its capacity for hyphal fusion and heterokaryon formation with its isogenic parent PH-1, the $m g v 1$ mutants lost this capacity.

\section{DISCUSSION}

The MGVI gene has all the characteristic features of MAP kinases and is closely related to the MPS1 gene of M. grisea. Like $M$. grisea mps 1 mutants, $F$. graminearum mgv 1 mutants are hypersensitive to cell wall degrading enzymes, suggesting a weaker cell wall in mutants in which the MAP kinase gene has been deleted. Intercalary or apical swollen bodies were formed on germ tubes cultured at an elevated incubation temperature (e.g., $32^{\circ} \mathrm{C}$ ) or in rich media. In $S$. cerevisiae, slt 2 mutants are defective in cell wall integrity and display enhanced sensitivity to cell wall degrading enzymes. On rich media such as YPD, slt2 mutants are unable to grow at $37^{\circ} \mathrm{C}$ in the absence of osmotic stabilizers (Lee et al. 1993). In Schizosaccharomyces pombe, spml mutants have cells of abnormal shape and are sensitive to elevated incubation temperature (Toda et al. 1996). In $C$.

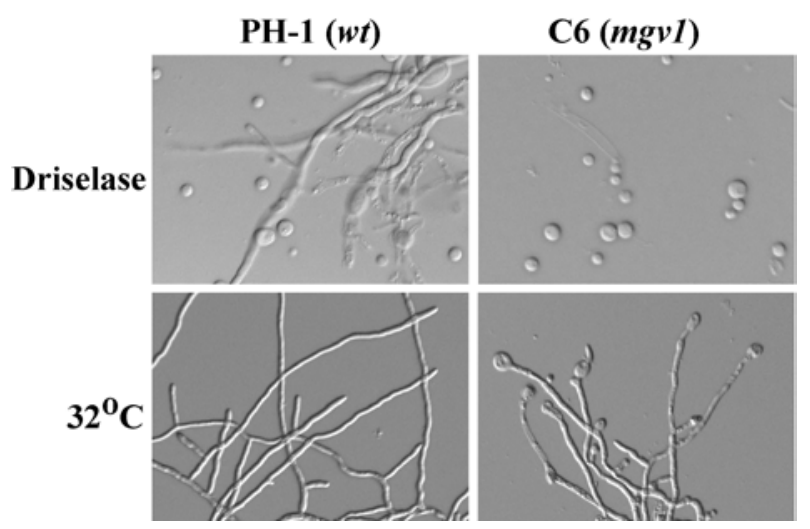

Fig. 5. Weakening of hyphal cell walls in mgvl mutants of Fusarium graminearum. After incubation for $30 \mathrm{~min}$ in protoplasting solution at $30^{\circ} \mathrm{C}$ (upper panels), the wild-type strain (PH-1) released protoplasts but still had many germlings remained intact. Germlings of the $m g v 1$ mutant (C6) were usually completely digested and released abundant protoplasts under the same conditions. When germlings grown in complete medium at $25^{\circ} \mathrm{C}$ were shifted to $32^{\circ} \mathrm{C}$, swollen and burst hyphal cells were observed in $\mathrm{C} 6$ but not in $\mathrm{PH}-1$. 
albicans, homozygous $m k c l$ deletion mutants are sensitive to high incubation temperature $\left(42^{\circ} \mathrm{C}\right)$, cell wall degrading enzymes, and antifungal compounds that interfere with 1,3-bglucan and chitin synthesis (Navarro-Garcia et al. 1998). Hyphal cells produced by $m k c l$ mutants are shorter than those of the wild type, and agar penetration by $m k c l$ hyphae is reduced on Spider medium (Navarro-Garcia et al. 1998). It is likely that the MGV1/MPS1 MAP kinase is well conserved in various fungi for regulating cell wall integrity and possibly nutrient sensing.

One intriguing phenomenon is that hyphal growth of $m g v 1$ mutants is reduced on solid media but normal in liquid media. The mutants have shortened aerial hyphae and form whitish, compact colonies. Similarly, in M. grisea, mps 1 mutants have been reported to form fewer aerial hyphae and conidia on oatmeal agar plates, while producing normal mycelial growth in liquid media (Xu et al. 1998). In Aspergillus nidulans, mpkA mutants also have a reduced growth rate and form fewer aerial hyphal (Bussink and Osmani 1999). In Botrytis cinerea, mutants disrupted in the MPS1 homologue also have significantly reduced aerial hyphae development, conidiation, and growth rate on solid media but grow normally in liquid media (L. Zheng and J.-R. Xu, unpublished data). Thus, this MAP kinase may be important for the development and elongation of aerial hyphae of $F$. graminearum and possibly other filamentous fungi. Modifications of cell wall structure and surface involved

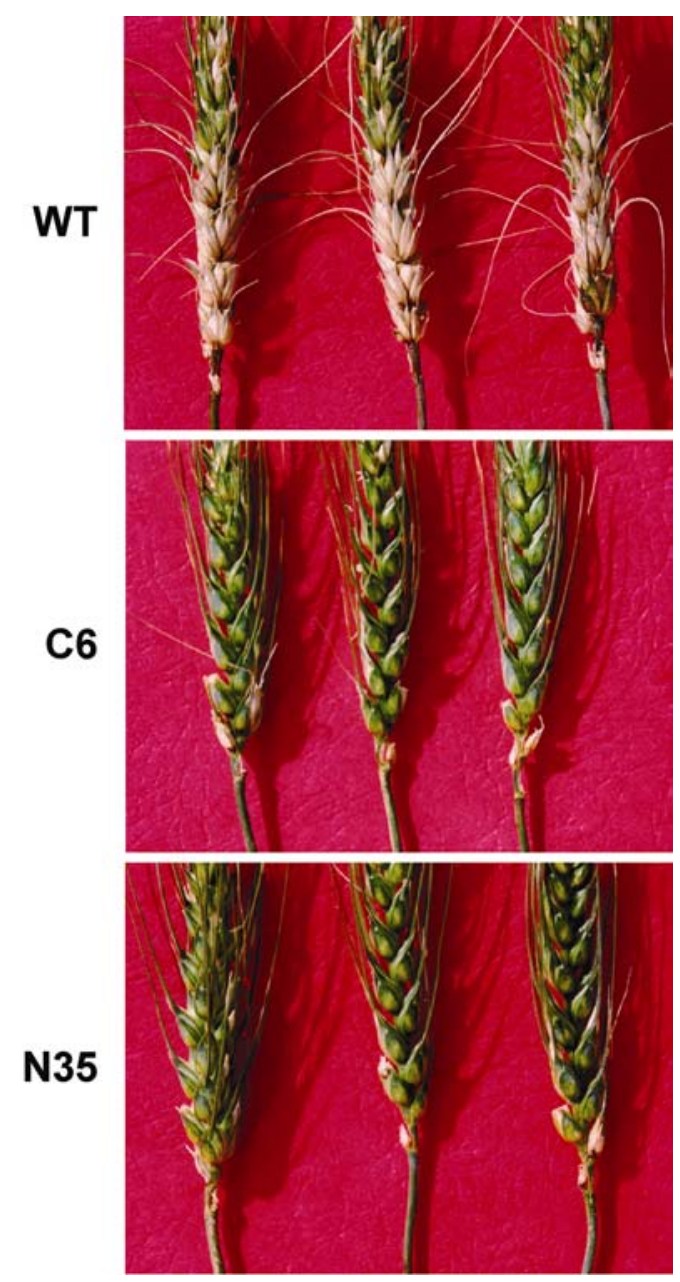

Fig. 6. Infection assay on wheat heads. Scab disease developed on flowering wheat heads inoculated in a basal spikelet with conidia from the wild-type strain PH-1 (top), mgv1 mutant C6 (middle), and mgvl mutant N35 (bottom). The photographs were taken 2 weeks after inoculation. In both mgvl mutants, the ability to cause necrosis beyond the point of inoculation was reduced. in aerial hyphae formation may be defective in the $m g v 1 \mathrm{mu}-$ tant. In M. grisea and B. cinerea, conidiation is also reduced in mutants that lack the MAP kinase gene, because conidiophores are produced on aerial hyphae. This is not the case in F. graminearum, which forms conidiophores in submerged cultures or very close to the surface on solid media. The $F$. graminearum mgvl mutant, like the $M$. grisea mps 1 mutant (Xu et al. 1998), is male-fertile but female-sterile. In F. graminearum, ascospores are thought to be important as primary inoculum and for long-distance dispersal (Trail and Common 2000). Chlamydospores are assumed to be one type of survival structure. It will be interesting to test whether the defects in female fertility and cell wall thickening have any effect on the survival and dispersal of the $m g v l$ mutants in the field.

Virulence is severely reduced in the $m g v l$ mutants. Because the vegetative growth rate is reduced on solid media, it is possible that the reduction in virulence of $m g v l$ mutants may be solely a consequence of the reduced growth rate. However, the growth rate of $m g v l$ mutants in liquid media is unaltered, and it is not clear whether growth conditions in planta may be more similar to solid media or liquid media. Furthermore, in many wheat heads inoculated with $m g v l$ conidia, symptoms were absent, or only the inoculated spikelets developed symptoms. These observations therefore may not be explained simply by a reduction in growth rate. Thus, the $m g v l$ mutants may be defective in producing some other virulence factors required for efficiently colonizing and spreading in wheat heads. As potent inhibitors of eukaryotic protein synthesis, trichothecenes may interfere with the induced resistance response of plants. DON production has been shown to contribute to the aggressiveness of $F$. graminearum in wheat (Proctor et al. 1995). The ability of $m g v l$ mutants to produce trichothecene mycotoxins in infected plants was greatly reduced (Table 3), and this may contribute to reduced levels of disease produced by these strains. In M. grisea, MPS1 is essential for appressorial penetration and infection of healthy rice leaves ( $\mathrm{Xu}$ et al. 1998). The MPS1 homologue is also important for pathogenicity in $B$. cinerea (Zheng and $\mathrm{Xu}$, unpublished data). In $C$. albicans, the homozygous $m k c l$ deletion mutants have reduced virulence in mice (Navarro-Garcia et al. 1998). In the obligate powdery mildew fungus Blumeria graminis, quantitative reverse transcription PCR indicated that the MPK2 gene, a homologue of $M G V 1$, is not expressed in ungerminated conidia, but its expression increases as germinated conidia form mature appressoria (Zhang and Gurr 2001), suggesting that MPK2 may be involved in plant penetration processes.

The wild-type $F$. graminearum, like many filamentous fungi, can undergo hyphal fusion between compatible isolates to form heterokaryons (Glass et al. 2000; Leslie 1993). Mutants generated from the same strain are usually compatible with each other because they have identical het loci, which determine vegetative compatibility. Interestingly, the $m g v l$ mutant is selfincompatible in heterokaryon formation. This phenotype has been reported as heterokaryon self-incompatibility (hsi) in other fungi (Correll et al. 1989; Glass et al. 2000). A single genetic locus, $h s i-1$, that determines heterokaryon self-incompatibility in Gibberella fujikuroi has been identified but not characterized (Correll et al. 1989). In Neurospora crassa, heterokaryon formation is delayed and reduced in the ham-l and ham-2 (hyphal anastomosis) mutants, which are defective in self- and non-self-hyphal fusions (Wilson and Dempsey 1999; Xiang et al. 2002). The HAM-2 gene encodes a protein that is highly conserved but of unknown function among eukaryotes (Xiang et al. 2002). Interestingly, ham-2 deletion mutants also have pleiotropic defects, including female sterility, shortened aerial hyphae, and reduced growth rate on solid medium (Xiang et al. 2002). It is possible that the MGVI MAP kinase pathway 
is somehow connected with $H A M-2$ and is involved in hyphal fusion and heterokaryon formation in filamentous fungi.

\section{MATERIALS AND METHODS}

\section{Strains and culture conditions.}

F. graminearum (lineage 7) wild-type strain $\mathrm{PH}-1$ (Trail and Common 2000) (NRRL 31084) and all the mutants generated in this study were routinely cultured at $25^{\circ} \mathrm{C}$ on $\mathrm{V} 8$ juice agar (Zheng et al. 2000). A nitl mutant 11622 of PH-1 was obtained from R. Bowden at Kansas State University. To compare growth rates on V8 juice, CM (Correll et al. 1987), TB3 (0.3\% yeast extract, $0.3 \%$ casamino acids, and $3 \%$ glucose), and $5 \times$ YEG (Zheng et al. 2000) agar media, the diameters of colonies formed by monoconidial cultures on $150 \times 15 \mathrm{~mm}$ petri plates were measured after incubation for 5 days. To assay conidiation, five conidia were introduced into $100 \mathrm{ml}$ of liquid CMC medium (Cappellini and Peterson 1965), the suspension was shaken at $250 \mathrm{rpm}$ for 5 days, and the concentration of conidia was determined with a hemacytometer. For dry weight measurements, $4.5 \times 10^{5}$ conidia were introduced into $100 \mathrm{ml}$ of liquid CMC or YEPD ( $0.3 \%$ yeast extract, $1 \%$ peptone, and $2 \%$ dextrose) medium in a 250 -ml flask, and the suspension was incubated at $25^{\circ} \mathrm{C}$ while being shaken. Mycelia were collected from 3-day-old cultures by filtration through two layers of Whatman no. 1 filter paper, baked at $100^{\circ} \mathrm{C}$ for 2 days, and then weighed. To assay degradation of cell walls and release of protoplasts, germlings collected from 14-h YEPD cultures were treated with Driselase $(20 \mathrm{mg} / \mathrm{ml})$ (Sigma, St. Louis, MO, U.S.A.) in $1.4 \mathrm{M} \mathrm{KCl}$ at $30^{\circ} \mathrm{C}$.

\section{DNA manipulations and sequence analysis.}

Standard molecular biology procedures were followed for gel electrophoresis and Southern blot analysis (Sambrook et al. 1989). Fungal DNA was extracted by the CTAB protocol as described previously (Proctor et al. 1995). Plasmid and cosmid DNA samples were purified with Qiagen plasmid preparation kits (Qiagen, Valencia, CA, U.S.A.) and sequenced with the BigDye Terminator kit (PE Applied Biosystems, Foster City, CA, U.S.A.). An ordered cosmid library consisting of 9,216 clones was constructed with PH-1 genomic DNA in the cosmid vector pMOcosX as described previously (Orbach 1994). RNA isolated from PH-1 mycelia starved for nitrogen was used to construct a cDNA library with a Lambda Uni-ZAP II cDNA synthesis kit (Stratagene, La Jolla, CA, U.S.A.) following the instructions provided by the manufacturer. Homologue searches of DNA/protein sequence databases were performed with the BLAST programs (Altschul et al. 1997). Amino acid sequence comparisons and alignments were made with the BESTFIT, PILEUP and BOXSHADE programs in the GCG Wisconsin software package (Accelrys, San Diego, CA, U.S.A.) and with Clustal X (Thompson et al. 1994).

Table 3. Trichothecene accumulation in wheat spikelets inoculated with mgv1 mutants

\begin{tabular}{lccc}
\hline & \multicolumn{3}{c}{ Trichothecene concentration $(\mathbf{p p m})^{\mathbf{a}}$} \\
\cline { 2 - 4 } Sample & DON & 3-A DON & 15-A DON \\
\hline N35-A & 0.9 & nd & nd \\
N35-B & 1.2 & nd & nd \\
C6-A & nd & nd & nd \\
C6-B & nd & nd & nd \\
N47-A & 431 & 6.7 & 285 \\
N47-B & 317 & 3.3 & 151 \\
\hline
\end{tabular}

${ }^{\mathrm{a}}$ Concentration of deoxynivalenol (DON), 3-acetyldeoxynivalenol (3-A DON) or 15-acetyldeoxynivalenol (15-A DON) in spikelet tissue, 14 days after inoculation. $\mathrm{nd}=$ not detectable $($ less than $0.5 \mathrm{ppm})$.

\section{Isolation of the $M G V 1$ gene.}

Degenerate PCR primers M3K1 (GGNGCNTAYGGNATHGTNTG) and M3K2 (ATYTCNGGNGCNCKRTACCA) were designed according to the conserved amino acid residues in fungal MAP kinases $S$. cerevisiae SLT2, Schizosaccharomyces pombe SPM1, and M. grisea MPS1 (Xu et al. 1998). These two primers were used to amplify the MPS1 homologue from $F$. graminearum by PCR consisting of 30 cycles of $1 \mathrm{~min}$ at $94^{\circ} \mathrm{C}, 1 \mathrm{~min}$ at $48^{\circ} \mathrm{C}$ and $2 \mathrm{~min}$ at $72^{\circ} \mathrm{C}$ followed by $5 \mathrm{~min}$ at $72^{\circ} \mathrm{C}$. First-strand cDNA synthesized with RNA isolated from nitrogen-starved PH-1 mycelia was used as the template. The PCR product amplified with $\mathrm{M} 3 \mathrm{~K} 1$ and $\mathrm{M} 3 \mathrm{~K} 2$, about $0.5 \mathrm{~kb}$, was cloned into the pGEM-T vector (Promega, Madison, WI, U.S.A.). The insert of a PCR clone homologous to MPS1 was amplified with PCR primers T7 and SP6 and used as the probe to screen the pMOcosX cosmid library and the Lambda ZAP II cDNA library constructed in this study. Seven MGV1-containing cosmid clones, including 5P24, 17O9, and 21E24, were isolated. The genomic sequence of the $M G V 1$ gene and its flanking regions was determined by primer walking. Two $M G V 1$ cDNA clones, $\mathrm{pC} 3 \mathrm{~K} 3$ and $\mathrm{pC} 3 \mathrm{~K} 4$, were also isolated and sequenced.

\section{Construction of $M G V 1$ gene replacement vector and mutants.}

The 3.4-kb EcoRI fragment containing the entire MGVI ORF was isolated from cosmid clone 1709 and cloned into the EcoRI site of the pBluescript KS (Stratagene) as pZMH4 (Fig. 2). The pZMH4 DNA was then digested with StuI and BglII and ligated with a 1.4-kb EcoRV-Bam HI fragment containing the bacterial hygromycin phosphotransferase gene $(h p h)$ from pAhd5 (Zheng et al. 2000). The resulting gene replacement vector, pZMH6, was linearized by digestion with Not I and transformed into the wild-type strain PH-1 as described previously (Proctor et al. 1995). Monoconidial cultures of transformants

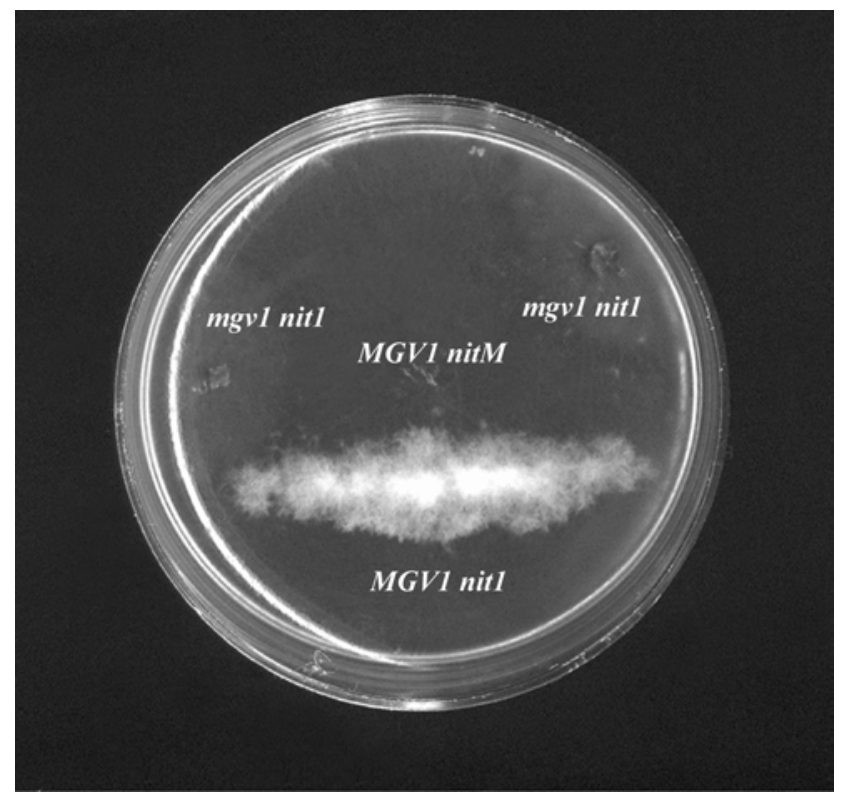

Fig. 7. Self-incompatibility in heterokaryon formation by strains of $F u$ sarium graminearum The petri plate contains minimal medium inoculated with four strains derived from the wild-type strain $\mathrm{PH}-1$ : the nitM mutant of PH-1 (MGV1 nitM) (center), C6 (mgv1 nit1) (upper left), N35 (mgv1 nit1) (upper right), and N47 (MGV1 nit1) (lower center). Only the $M G V 1$ strains formed a heterokaryon characterized by the regeneration of wild-type growth due to complementation of auxotrophic mutations. The $m g v 1$ mutants were unable to form heterokaryons with isogenic $M G V 1$ strains. 
resistant to hygromycin B (Calbiochem, La Jolla, CA, U.S.A.) at a concentration of $250 \mu \mathrm{g} / \mathrm{ml}$ on $\mathrm{V} 8$ juice agar plates were screened by PCR with primers $17 \mathrm{~F}$ (TGGGTTGACCTGATAATG) and H855R (GCTGATCTGACCAGTTGC). The primer $17 \mathrm{~F}$ sequence is located in the upstream region of MGVI and is not included in pZMH6. Only transformants with homologous recombination between pZMH6 and the endogenous $M G V 1$ gene will have the 450-bp PCR product. Putative $m g v 1$ deletion mutants identified in the PCR screens were further confirmed by Southern blot analysis. For complementation assays, the wild-type $M G V 1$ gene was reintroduced into the mgv1 mutant C6 by co-transformation with pSM334 (kindly provided by Seogchan Kang at Pennsylvania State University), a vector containing the neomycin phosphotransferase fused to the promoter of the Cochliobolus heterostrophus glyceraldehyde-3-phosphate dehydrogenase gene (Van Wert and Yoder 1992). Neomycin-resistant transformants were selected on CM containing $200 \mu \mathrm{g}$ of neomycin per ml (Amresco, Solon, $\mathrm{OH}$, U.S.A.) and screened by PCR with primers K1R (GAGTGGATGTACTTGAGA) and 13F (ATACACCACCACACAAATAC).

\section{Infection assays on flowering wheat heads.}

Conidia were collected from 5-day-old CMC cultures by filtration through two layers of Miracloth (Calbiochem) and resuspended in sterile distilled water to a concentration of $0.5 \times$ $10^{5}$ to $1 \times 10^{5}$ conidia/ml. Approximately 6 -week-old plants of the wheat cultivars Norm and Pioneer 2375 with flowering heads were used for infection assays. A floret in the basal spikelet of the flowering wheat head was injected with $10 \mu \mathrm{l}$ of the conidial suspension. Inoculated plants were placed in a humidity chamber for 3 days and then transferred to a greenhouse with normal growth conditions. Symptomatic spikelets in each head were counted 14 days after inoculation. For the co-inoculation assay, conidia from PH-1 and C6 were mixed in equal ratios and used to inoculate flowering wheat heads. Analysis of variance was used to compare the mean number of symptomatic spikelets. As the wheat cultivar had no significant effect on the mean number of symptomatic spikelets $(P=0.74)$, results from both cultivars were pooled. The number of inoculated wheat heads per treatment ranged from 15 to 18 . All tests were repeated once.

\section{Sexual reproduction and vegetative incompatibility assays.}

Perithecium formation in selfing and outcrossing cultures was assayed on modified carrot agar plates as described previously (Bowden and Leslie 1999). Ascospore cirri from perithecia on outcross plates, inoculated with C6 and 11622, were collected and resuspended in distilled water. Random ascospores were analyzed for resistance to hygromycin and $1 \%$ potassium chlorate on minimal medium. The nit1 mutants of C6, N35, and N47 and a nitM mutant of PH-1 were obtained as described previously (Correll et al. 1987). To assay vegetative incompatibility, strains with complementary auxotrophic mutations were paired on minimal medium in $60 \times 15 \mathrm{~mm}$ petri plates. The plates were incubated at ambient temperature under continuous fluorescent and near-UV light and allowed to grow together for 3 weeks as described previously (Correll et al. 1987).

\section{Measurement of DON production.}

The directly inoculated spikelets from five plants per treatment were pooled for DON analysis. The treatments were repeated once. Trichothecene levels in infected tissues were determined according to the method of Mirocha et al. (1998) with modifications. Briefly, $1 \mathrm{~g}$ of the pooled inoculated spikelets was extracted with $10 \mathrm{ml}$ of a mixture of acetonitrile and water $(84: 16 \mathrm{v} / \mathrm{v})$ in a $50-\mathrm{ml}$ centrifuge tube for $1 \mathrm{~h}$. After column cleanup, $1 \mathrm{ml}$ of the extract was placed in a 0.5 -dram vial and evaporated to dryness under nitrogen. The sample was derivatized with $20 \mu \mathrm{l}$ of trimethylsilyl ether (TMS) reagent (TMSI/ TMCS 100:1, Sigma) and diluted with $200 \mu \mathrm{l}$ of isooctane. Gas chromatography-mass spectroscopy (GC-MS) sample analysis was carried out on a Varian Saturn 3 GC-MS system with a Varian 3400 CX gas chromatograph and 8200 CX AutoSampler. The quantitative analysis was done in selected ion monitoring mode. Ions at $\mathrm{m} / \mathrm{z}$ 235.10, 259.10, 295.10, 392.15, and 412 were monitored. A full-scan GC-MS analysis (with a scan range of $\mathrm{m} / \mathrm{z}, 180$ to 440) was carried out for toxin identification in a wheat head sample. The gas chromatograph column was a J\&W DB-5ms, $30 \mathrm{~m}$ in length, with $0.25-\mu \mathrm{m}$ film thickness and $250-\mu \mathrm{m}$ i.d. The GC temperature program was an initial temperature of $150^{\circ} \mathrm{C}$ for $1 \mathrm{~min}$, ramped to $280^{\circ} \mathrm{C}$ at $25^{\circ} \mathrm{C} / \mathrm{min}$, followed by isothermal conditions for $5 \mathrm{~min}$.

\section{ACKNOWLEDGMENTS}

We thank Larry Dunkle and Gregory Shaner at Purdue University for critical reading of this manuscript. We also thank Frances Trail at Michigan State University and Robert Bowden at Kansas State University for providing PH-1 and its nit mutants, and Rubella Goswami for her help in trichothecene measurement. This work was supported by a grant from the U.S. Wheat and Barley Scab Initiative to J.X. and H.C.K. This is journal article no. 16853 of the Purdue University Agricultural Experiment Station.

\section{LITERATURE CITED}

Altschul, S. F., Madden, T. L., Shaffer, A. A., Zhang, Z., Miller, W., and Lipman, D. J. 1997. Gapped BLAST and PSI-BLAST: A new generation of protein database search programs. Nucleic Acids Res. 25:3389-3402.

Booth, C. 1971. The Genus Fusarium. Commonwealth Mycological Institute, Kew, England.

Bowden, R. L., and Leslie, J. F. 1999. Sexual recombination in Gibberella zeae. Phytopathology 89:182-188.

Bussink, H., and Osmani, S. A. 1999. A mitogen-activated protein kinase (MPKA) is involved in polarized growth in the filamentous fungus Aspergillus nidulans. FEMS Microbiol. Lett. 173:117-125.

Cappellini, R. A., and Peterson, J. L. 1965. Macroconidium formation in submerged cultures by a non-sporulating strain of Gibberella zeae. Mycologia 57:962-966.

Correll, J. C., Klittich, C. J. R., and Leslie, J. F. 1987. Nitrate nonutilizing mutants of Fusarium oxysporum and their use in vegetative compatibility tests. Phytopathology 77:1640-1646.

Correll, J. C., Klittich, C. J. R., and Leslie, J. F. 1989. Heterokaryon selfincompatibility in Gibberella fujikuroi (Fusarium moniliforme). Mycol. Res. 93:21-27.

Desjardins, A. E., Hohn, T. M., and McCormick, S. P. 1993. Trichothecene biosynthesis in Fusarium species: Chemistry, genetics, and significance. Microbiol. Rev. 57:595-604.

Diez-Orejas, R., Molero, G., Navarro-Garcia, F., Pla, J., Nombela, C., and Sanchez-Perez, M. 1997. Reduced virulence of Candida albicans $M K C 1$ mutants: A role for mitogen-activated protein kinase in pathogenesis. Infect. Immun. 65:833-837.

Francl, L., Shaner, G., Bergstrom, G., Gilbert, J., Pedersen, W., DillMacky, R., Sweets, L., Corwin, B., Jin, Y., Dallenberg, D., and Wiersma, J. 1999. Daily inoculum levels of Gibberella zeae on wheat spikes. Plant Dis. 83:662-666.

Gerlach, W., and Nirenberg, H. I. 1982. The Genus Fusarium-A Pictorial Atlas. Mitt. Biol. Bundesanst. Land- Forstwirtsch., BerlinDahlem, 209.

Glass, N. L., Jacobson, D. J., Patrick K. T., and Shiu, P. K. T. 2000. The genetics of hyphal fusion and vegetative incompatibility in filamentous ascomycete fungi. Annu. Rev. Genet. 34:165-86.

Lee, K. S., Irie, K., Gotoh, Y., Watanabe, Y., Araki, H., Nishida, E., Matsumoto, K., and Levin, D. E. 1993. A yeast mitogen-activated protein kinase homologue (Mpk1p) mediates signaling by protein kinase $\mathrm{C}$. Mol. Cell. Biol. 13:3067-3075.

Leslie, J. F. 1993. Fungal vegetative compatibility. Annu. Rev. Phytopathol. 31:127-150.

McMullen, M., Jones, R., and Gallenberg, D. 1997. Scab of wheat and barley: A re-emerging disease of devastating impact. Plant Dis. 81: 1340-1348.

Mirocha, C. J., Kolaczkowski, E., Xie, W., Yu, H., and Jelen, H. 1998. 
Analysis of deoxynivalenol and its derivatives (batch and single kernel) using gas chromatography/mass spectrometry. J. Agric. Food Chem. 46:1414-1418.

Navarro-Garcia, F., Alonso-Monge, R., Rico, H., Pla, J., Sentandreu, R. and Nombela, C. 1998. A role for the MAP kinase gene $M K C 1$ in cell wall construction and morphological transitions in Candida albicans. Microbiology 144:411-424.

Nelson, P. E., Toussoun, T. A., and Marasas, W. F. O. 1983. Fusarium Species: An Illustrated Manual for Identification. Pennsylvania State University Press, University Park.

O’Donnell, K., Kistler, H. C., Tacke, B. K., and Casper, H. H. 2000. Gene genealogies reveal global phylogeographic structure and reproductive isolation among lineages of Fusarium graminearum, the fungus causing wheat scab. Proc. Natl. Acad. Sci. USA 97:7905-7910.

Orbach, M. J. 1994. A cosmid with a hygromycin resistance marker for fungal library construction and screening. Gene 150:159-162.

Parry, D. W., Jenkinson, P., and McLeod, L. 1995. Fusarium ear blight (scab) in small grain cereals-A review. Plant Pathol. 44:207-238.

Proctor, R. H., Hohn, T. M., and McCormick, S. P. 1995. Reduced virulence of Gibberella zeae caused by disruption of a trichothecene toxin biosynthetic gene. Mol. Plant-Microbe Interact. 8:593-601.

Sambrook, J., Fritsch, E. F., and Maniatis, T. 1989. Molecular Cloning: A Laboratory Manual. 2nd ed. Cold Spring Harbor Laboratory, Cold Spring Harbor, NY.

Sutton, J. C. 1982. Epidemiology of wheat head blight and maize ear rot caused by Fusarium graminearum. Can. J. Plant Pathol. 4:195-209.

Thompson, J., Higgins, D., and Gibson, T. 1994. Clustal W: Improving the sensitivity of progressive multiple sequence alignment through sequence weighting, positions-specific gap penalties and weight matrix choice. Nucleic Acids Res. 22:4673-4680.

Toda, T., Dhut, S., Superti-Furga, G., Gotoh, Y., Nishida, E., Sugiura, R., and Kuno, T. 1996. The fission yeast $p m k 1^{+}$gene encodes a novel mitogen-activated protein kinase homolog which regulates cell integ- rity and functions coordinately with the protein kinase $\mathrm{C}$ pathway. Mol. Cell. Biol. 16:6752-6764.

Trail, F., and Common, R. 2000. Perithecial development by Gibberella zeae: A light microscopy study. Mycologia 92:130-138.

Trail, F., Xu, H., Loranger, R., and Gadoury, D. 2002. Physiological and environmental aspects of ascospore discharge in Gibberella zeae (anamorph Fusarium graminearum). Mycologia 94:181-189.

Van Wert, S. L., and Yoder, O. C. 1992. Structure of the Cochliobolu heterostrophus glyceraldehyde-3-phosphate dehydrogenase gene. Curr. Genet. 22:29-35.

Wearing, A. H., and Burgess, L. W. 1977. Distribution of Fusarium roseum 'Graminearum' group 1 and its mode of survival in eastern Australian wheat belt soils. Trans. Br. Mycol. Soc. 69:429-442.

Wilson, J. F., and Dempsey, J. A. 1999. A hyphal fusion mutant in Neurospora crassa. Fungal Genet. Newsl. 46:31.

Windels, C. E. 2000. Economic and social impacts of Fusarium head blight: Changing farms and rural communities in the northern Great Plains. Phytopathology 90:17-21.

Xiang, Q., Rasmussen, C., and Glass, N. L. 2002. The ham-2 locus, encoding a putative transmembrane protein, is required for hyphal fusion in Neurospora crassa. Genetics 160:169-180.

$\mathrm{Xu}$, J. R. 2000. MAP kinases in fungal pathogens. Fungal Genet. Biol 31:137-152.

Xu, J.-R., Staiger, C. J., Hamer, J. E. 1998. Inactivation of the mitogenactivated protein kinase MPS1 in the rice blast fungus prevents penetration of host cells but allows activation of plant defense responses. Proc. Natl. Acad. Sci. USA 95:12713-12718.

Zhang, Z., and Gurr, S. J. 2001. Expression and sequence analysis of the Blumeria graminis mitogen-activated protein kinase genes, $m p k 1$ and $m p k 2$. Gene 266:57-65.

Zheng, L., Campbell, M., Murray, J., Lam, S., and Xu, J. R. 2000. The $B M P 1$ gene is essential for pathogenicity in the gray mold fungus $B o$ trytis cinerea. Mol. Plant-Microbe Interact. 13:724-732. 